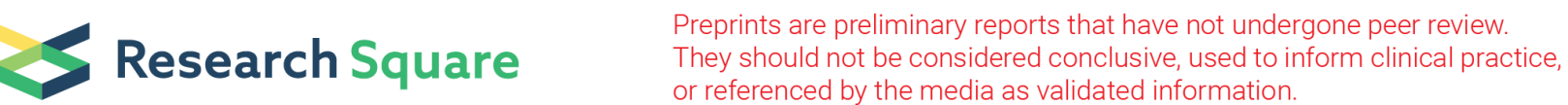

\section{Treatment effects of pharmacotherapy and non- pharmacotherapy of Alzheimer dementia: A systematic review overview and updated network meta-analysis.}

Chitima Boongird ( $\nabla$ Chitima.amo@mahidol.ac.th )

Mahidol University Faculty of Medicine Ramathibodi Hospital https://orcid.org/0000-0002-7594-2460

Worapong Tearneukit

Somdet Chaopraya Hospital: Somdet Chaopraya Institute of Psychiatry

Thunyarat Anothaisintawee

Mahidol University Faculty of Medicine Ramathibodi Hospital

Gareth McKay

Queen's University Belfast

Ammarin Thakkinstian

Mahidol University Faculty of Medicine Ramathibodi Hospital

\section{Protocol}

Keywords: Alzheimer dementia, pharmacotherapy, non-pharmacotherapy, network meta-analysis

Posted Date: October 1st, 2021

DOI: https://doi.org/10.21203/rs.3.rs-940193/v1

License: (c) (1) This work is licensed under a Creative Commons Attribution 4.0 International License.

Read Full License 


\section{Abstract}

Introduction: Many existing systematic reviews and meta-analyses summarize the evidence of pharmacotherapies (PTs) and non-pharmacotherapies (NPTs) for wide range of available interventions for the management of Alzheimer dementia (AD). However, a succinct summary and efficacy comparison across the different types of treatments and interventions is lacking. We aim to identify the treatment or treatments with the highest efficacy of current pharmacological and non-pharmacological treatments for $A D$.

Methods and analysis: Systematic reviews of randomized controlled trials (RCTs) will be selected according to the following criteria: conducted in elderly patients aged 60 years or older with AD, applied pairwise meta-analysis (PMA) or network meta-analysis (NMA) for pooled relative treatment effects for at least one pair of PTs and NPTs, and at least one of following outcomes was provided for patients/caregivers: cognitive function status, behavior, quality of life. The summary characteristics for each RCT will include the general design, participants, intervention, outcome measurements, duration of therapy and adverse events. The risk of bias will be assessed using Risk of Bias in Systematic Reviews (ROBIS) checklist and the risk of bias of individual RCTs will be assessed using the Revised Cochrane risk-of-bias tool.

Ethics and Dissemination: Ethical approval will not be required because this study is based solely on published literature that meets accepted ethical standards, and there will be no concerns about privacy. Dissemination of the evidence obtained will include publication in a peer-reviewed international journal to improve clinical practices on the basis of robust scientific evidence.

PROSPERO registration number: CRD42021228245

\section{Article Summary}

\section{Strengths and Limitations}

- This is an umbrella systematic review protocol to summarize the evidence for pharmacological and non-pharmacological treatments for Alzheimer dementia.

- The protocol has been developed according to the published PRISMA-P guidelines.

- We aim to identify the treatment or treatments with the highest efficacy of current pharmacological and non-pharmacological treatments for $A D$ based on cognitive function status, behaviors, and quality of life for both patients and caregivers.

- The main limitation of this study is the limited sample size for non-pharmacotherapies (NPTs) and the substantial variation in characteristics of the interventions. The authors of the primary studies reported different conceptual frameworks for the interventions which could be broad with quite general descriptions. 


\section{Background}

Dementia is an age-related neurodegenerative condition with increased prevalence in older populations. The World Health Organization (WHO) estimated there were more than 50 million people with dementia, with approximately 60 percent originating from low and middle-income countries ${ }^{1}$. Almost 600,000 individuals were reported with dementia in Thailand in 2015, a number anticipated to exceed one million by $2030^{2}$. The familial, societal and economic burdens associated with this condition is significant with a recent survey indicating $86 \%$ of dementia caregivers in Thailand were from an informal household source with the bulk of associated care costs characterized as indirect due to lost productivity of unpaid caregivers ${ }^{2}$.

Multiple systematic reviews with pairwise meta-analysis (PMA) and network meta-analysis (NMA) $)^{3-8}$, including several Cochrane Collaborations, have identified pharmacological treatments (PT) to maintain or reduce the rate of decline in cognitive function in older adults with early-stage dementia. Several reviews 589 have recommended the use of acetylcholinesterase inhibitors (AChEI) such as donepezil, rivastigmine, and galantamine, in addition to memantine, an uncompetitive antagonist of N-methyl-Daspartate (NMDA) glutamate receptors, in moderate to severe dementia. In addition, herbal remedies have included huperzine $A^{10}$, ginkgo biloba leaf $(E G b 761)^{11}$, and traditional Chinese medicines ${ }^{12}$ that have demonstrated clinical benefits on cognitive function similar to AChEl. However, reported adverse effects and the high risk of contraindications associated with $\mathrm{PTs}{ }^{13}$, have led to increasing acceptance of nonpharmacological treatments (NPT) for limiting dementia progression. The most common NPTs include cognitive stimulation (CST), cognitive rehabilitation (CR), music therapy (MT), computerized cognitive training (CCT), and non-invasive brain stimulation (repetitive trans magnetic stimulation (rTMS)) have been associated with improved cognitive function and associated behaviors such as decreased agitation and depressed mood ${ }^{14-19}$.

Currently, no disease-modifying treatment has proven fully effective in controlling the symptoms of Alzheimer's dementia (AD). Despite focused research efforts on the preclinical and early stage of $A D$, and the development of new disease-modifying treatments to reduce the pathophysiological changes associated with $A D^{20}$ (i.e. amyloid deposition, phosphorylation and protein aggregation), no significant clinical effect has been achieved, to date ${ }^{21}$. Caring for people with $A D$ and dementia requires the involvement of a large number of healthcare professionals (eg. psychologists, occupational therapists, etc.) and caregivers to provide a comprehensive and individualized management plan. NPTs tend to be non-invasive, safe and with few side effects with an aim to maintain cognitive function for as long as possible as the disease progresses, thus reducing disability and improving patients and caregivers' quality of life.

Despite the increasing evidence of the benefits associated with PTs and NPTs, published comparisons between both have been limited. In addition, implementation of real-world evidence remains challenging given the financial and resource limitations in the majority of healthcare settings. As such, this study will 
provide an overview and efficacy comparisons between individual PTs and NPTs for the treatment of AD characterized by cognitive function status, behaviors, and quality of life (QoL) for both patients and caregivers. A NMA will be applied to update and estimate treatment effects for all regimens, ranking each against the outcomes indicated. The findings from this umbrella review will provide a platform for future research design and cost effectiveness comparisons of pharmacological and non-pharmacological dementia treatments in real practice settings, where resources are limited.

\section{Research question:}

Do the effects of PTs and NPTs differ in the outcomes for Alzheimer dementia patients?

\section{Patient and public involvement}

No patients were involved in the design of this study. We will disseminate plain language summaries to relevant patient groups.

\section{Objective}

- To estimate and summarize relative treatment effects between individual PTs and NPTs

- To rank PT and NPT according to improved cognitive function status, behaviors, and QoL for patients and caregivers

\section{Methods And Design}

This overview study and NMA update will be conducted in accordance with PRISMA guidelines for network meta-analysis and the protocol was registered with the International Prospective Register of Systematic Reviews (PROSPERO) in February 2021 (registration number CRD42021228245)

\section{Study selection process}

\section{Type of study}

Systematic reviews of randomized controlled trials (RCTs) will be selected according to the following criteria:

- Conducted in elderly patients aged 60 years or older with $A D$

- Reported PMA or NMA for pooled relative treatment effects for at least one pair of PTs or NPTs

- Provided at least one of the following outcomes for patients/caregivers: cognitive function status, rate of falls, behavior, QoL. 
Individual RCTs included in selected systematic reviews will be re-selected based on the following criteria:

- Undertaken in elderly patients aged 60 years or older with AD

- Compared relative treatment effect of at least one pair of PTs or NPTs

- Provided at least one of the following outcomes for patients/caregivers: cognitive function status, rate of falls, behavior, QoL.

The individual RCTs will be excluded where insufficient data is available for pooling following 2-3 attempts to contact the corresponding author.

\section{Type of Participants}

We will include any RCT that included elderly participants aged 60 years or older diagnosed as AD using their country's standard AD diagnostic criteria, e.g., Diagnostic and Statistical Manual of Mental Disorders (DSM) IV ${ }^{22}$ or DSM-5 ${ }^{23}$ (American Psychiatric Association (APA)), the National Institute of Neurological and Communicative Disorders and Stroke-Alzheimer Disease and Related Disorders Association, and/or National Institute on Aging (NIA) and the Alzheimer's Association criteria ${ }^{24}$, and/or the International Working Group, and neuropsychological tests to diagnose AD. We will use the MiniMental State Examination (MMSE) or Montreal Cognitive Assessment/Clinical Dementia Rating Scale for additional screening.

\section{Intervention}

Interventions of interest include any comparison between individual NPTs and usual/standard care or PTs and placebo or head-to-head comparison between PTs and PTs:

\section{NPTs}

The most common NPTs include CST, CR, MT, CCT, and non-invasive brain stimulation (rTMS) and the following will be considered:

1. CST

2. $\mathrm{CR}$

3. Reminiscence therapy (RMT)

4. CCT

5. Physical activity (PA)

1. Aerobic only

2. Non-aerobic

3. Combination or multicomponent

4. High intensity functional exercise 
6. Functional-based analysis intervention (behavioral management)

7. Occupational therapy-fall prevention program

8. Care-giver interventions

9. Massage

10. Recreation therapy

11. MT

12. Aromatherapy

13. Psychological therapy

14. Social intervention

15. Multisensory stimulation (MSS or Snoezelen)

16. Light therapy

17. Art therapy

18. Therapeutic touch

19. Multicomponent therapy (SONAS)

20. Acupuncture

21. Non-invasive brain stimulation (rTMS, transcranial direct current stimulation (tDCS))

22. Animal assisted therapy (AAT)

23. Usual/ standard care

24. PTs

1. Cognitive enhancer

1. Cholinesterase inhibiter (ChEls); donepezil, galantimine, rivastigmine

2. NMDA receptor antagonists; memantine

3. Herbs; Huperzia serrate, Gingo biloba extract (EGB 761) Yokukansan (TJ-54), Panax Ginseng, saffron (crocus sativus L.)

4. Melatonin

25. Placebo

\section{Outcome measure}

The outcomes of interest include cognitive function, behavioral and psychological symptoms of dementia (BPSD), QoL, functional status and rate of falls. Cognitive function assessed in the original studies mainly using the Mini-Mental State Examination (MMSE), Alzheimer's Disease Assessment-Scale Cognitive (ADAS-Cog), Montreal Cognitive Assessment (MoCA), Global Cognitive Functions (e.g., Clock drawing test), Clinical Global Impression of Change (CGIC), Clinician's Interview-Based Impression of Change (CIBIC), self or caregiver reported functions (caregiver input), Clinical Dementia Rating (CDR). The BPSD were evaluated by neuropsychiatric inventory (NPI), brief psychiatric rating scales and other 
behavior assessment tools. QoL and functional status were measured by EQ5D, Barthel or modified Barthel index. Rate of falls was the number of reported falls. A fall was defined as "a person unintentionally coming to rest on the ground, floor or other level." The secondary outcomes include caregiver burden and QoL measured by Zarit burden inventory scale (ZBI) and EQ5D, respectively.

\section{Search strategy}

Relevant systematic reviews and meta-analyses will be identified by a single reviewer (CB) from PubMed and Scopus from initiation to January 2021. The search terms and strategies are based on patients $(P)$, interventions/ comparators $(\mathrm{I} / \mathrm{C})$, outcomes $(\mathrm{O})$, and study design $(\mathrm{S})$ as follows:

P: "Alzheimer dementia; I/C: donepezil, rivastigmine, galantamine, memantine, huperzine-A, EGb761, Yokukansan (TJ-54), "cognitive stimulation therapy", "cognitive rehabilitation", "music therapy", "physical activity", "non-invasive brain stimulation", "non-pharmacological therapy"; 0: cognitive, behaviors, function, "quality of life"; S: meta-analysis.

Searches will be updated every 3 months until December 2021.

\section{Data extraction}

Data will be extracted independently by two reviewers (CB and WT). Data for systematic reviews will be extracted including systematic review characteristics (pairwise or network meta-analysis), number of RCTs included, number of interventions/comparators, type of outcomes, methods used for pooling relative treatment effects (standardized/unstandardized mean difference (SMD/UMD) for continuous outcomes; ORs, RRs for dichotomous outcomes). The following data will be extracted from individual RCTs: patient characteristics (e.g., age, percentage female, body mass index, diabetes, hypertension, cardiovascular disease status etc.), intervention/comparator, type of outcomes and tool/scale used to determine outcome measures. In addition, data for pooling will be extracted including the number of patients, mean and standard deviation (SD) by intervention groups for continuous outcomes, contingency data of intervention and dichotomous outcomes.

\section{Risk of bias and quality assessment}

Risk of bias will be assessed using the Risk of Bias in Systematic Reviews (ROBIS) checklist ${ }^{25}$ by two independent reviewers (CB and TA), disagreement will be resolved by a third reviewer (AT). Four domains will be considered including study eligibility criteria, methods used to identify and/or select studies, data collection and appraisal of studies, and synthesis/findings. The result will be graded as low or high risk of bias if there is sufficient information to assess, otherwise, it will be graded as unclear.

Risk of bias of individual RCTs will be assessed using the Revised Cochrane risk-of-bias tool for randomized trials considering the five domains of randomization, deviation from the intended intervention, missing outcome data, outcome measurements, and selection of the reported results. Each domain will be rated as low, some concerns, and high risk according to the risk-of-bias tool algorithm. The 
RCT will be judged as high risk of bias if at least one domain is rated high risk; low risk of bias if all domains are rated as low risk; otherwise, the RCT will be judged as some concerns. Any disagreement will be resolved by consensus or judgment of a third reviewer (AT).

\section{Data Synthesis}

A two-stage NMA will be performed as follows: Relative treatment effects (e.g., SMD, UMD, In ORs/In RR) along with variance estimates for individual RCTs. Relative treatment effects will be pooled across RCTs using a consistency model and all possible relative treatment comparisons estimated. Consistency assumptions will be assessed using design-treatment interaction methods with a loop-specific approach to identify inconsistencies, where present. Patient characteristics associated with specific loops will be explored and treatment efficacy ranked for each outcome using surface under cumulative ranking curves (SUCRA). Adjusted funnel plots will assess publication bias.

We will perform subgroup analysis to explore source/s of heterogeneity and inconsistency, and will focus on factors including extreme elderly patients (age 85 years or older), stage of disease as defined by the functional assessment staging test (FAST) criteria $^{26}$, etc. In addition, a sensitivity analysis will be performed by excluding studies with high risk of bias, very old age, low/high effect size/variance (e.g., $25^{\text {th }}$ or $75^{\text {th }}$ percentile) to determine the robustness of the findings. All analyses will be performed using STATA 16.

\section{Discussion}

Currently, a comprehensive review of the comparison of pharmacological and non-pharmacological treatments to determine the most efficacious and cost-effective options for AD patients is lacking. Despite evidence supporting small to medium ranged benefits of pharmacological treatments, the majority of people with dementia have limited choice of therapies due to costs and associated intolerability of medications. In addition, non-pharmacological treatments require multidisciplinary specialized teams which are often limited in real world clinical settings. This umbrella review and network meta-analysis will fill the knowledge gap with findings to guide evidence-based decision-making in AD treatment strategies to support recommended AD management, not only for patients but also for family caregivers.

\section{Declarations}

Acknowledgements We would like to thank Ramathibodi research center to support useful information for continuing this study.

Competing of interest none declared

\section{Ethics and dissemination}


Ethical approval will not be required because this study is based solely on published literature that meets accepted ethical standards, and there will be no concerns regarding privacy. The evidence obtained will be disseminated through peer-reviewed publication to inform clinical practices with scientific evidence.

Author statement $\mathrm{CB}$ and $\mathrm{AT}$ are co-first authors and contributed equally to the protocol. GMK provided input on the design and drafting of the protocol. WT and TA are the co-corresponding authors. All authors read and approved the final protocol.

Funding This research received no specific grant from any funding agency in the public, commercial or not-for-profit sectors.

\section{References}

1. Associations As. Alzheimer's Disease Facts \& Figures. WHO report. 2018 [Available from: https://www.who.int/news-room/fact-sheets/detail/dementia accessed Febuary, 222021.

2. Chuakhamfoo NN, Phanthunane P, Chansirikarn S, et al. Health and long-term care of the elderly with dementia in rural Thailand: a cross-sectional survey through their caregivers. BMJ Open. 2020;10(3):e032637. doi:10.1136/bmjopen-2019-032637 [published Online First: 2020/03/27].

3. Di Santo SG, Prinelli F, Adorni F, et al. A meta-analysis of the efficacy of donepezil, rivastigmine, galantamine, and memantine in relation to severity of Alzheimer's disease. J Alzheimers Dis. 2013;35(2):349-61. doi:10.3233/JAD-122140 [published Online First: 2013/02/16].

4. Kobayashi $H$, Ohnishi T, Nakagawa R, et al. The comparative efficacy and safety of cholinesterase inhibitors in patients with mild-to-moderate Alzheimer's disease: a Bayesian network meta-analysis. Int J Geriatr Psychiatry. 2016;31(8):892-904. doi:10.1002/gps.4405 [published Online First: 2015/12/19].

5. Dou KX, Tan MS, Tan CC, et al. Comparative safety and effectiveness of cholinesterase inhibitors and memantine for Alzheimer's disease: a network meta-analysis of 41 randomized controlled trials. Alzheimers Res Ther. 2018;10(1):126. doi:10.1186/s13195-018-0457-9 [published Online First: 2018/12/29].

6. Tricco AC, Ashoor HM, Soobiah C, et al. Comparative Effectiveness and Safety of Cognitive Enhancers for Treating Alzheimer's Disease: Systematic Review and Network Metaanalysis. J Am Geriatr Soc. 2018;66(1):170-78. doi:10.1111/jgs.15069 [published Online First: 2017/11/14].

7. Tsoi KKF, Chan JYC, Chan FCH, Hirai HW, Kwok TCY, Wong SYS. Monotherapy is Good Enough for Patients With Mild-to-Moderate Alzheimer's Disease: A Network Meta-Analysis of 76 Randomized Controlled Trials. Clin Pharmacol Ther. 2019;105(January):121-30.

8. Guo J, Wang Z, Liu R, et al. Memantine, Donepezil, or Combination Therapy-What is the best therapy for Alzheimer's Disease? A Network Meta-Analysis. Brain Behav. 2020;10(11):e01831. doi:10.1002/brb3.1831 [published Online First: 2020/09/12]. 
9. Chen R, Chan PT, Chu H, et al. Treatment effects between monotherapy of donepezil versus combination with memantine for Alzheimer disease: A meta-analysis. PLoS One. 2017;12(8):e0183586. doi:10.1371/journal.pone.0183586 [published Online First: 2017/08/23].

10. Liang J, Li J, Jia R, et al. Identification of the optimal cognitive drugs among Alzheimer's disease: a Bayesian meta-analytic review. Clin Interv Aging. 2018;13:2061-73. doi:10.2147/CIA.S184968 [published Online First: 2018/11/15].

11. Thancharoen O, Limwattananon C, Waleekhachonloet O, et al. Ginkgo biloba Extract (EGb761), Cholinesterase Inhibitors, and Memantine for the Treatment of Mild-to-Moderate Alzheimer's Disease: A Network Meta-Analysis. Drugs Aging. 2019;36(5):435-52. doi:10.1007/s40266-019-00648-x [published Online First: 2019/04/03].

12. May BH, Feng M, Hyde AJ, Hugel H, Chang S, Dong L, Gou X, Zhang AL, Lu C, Xue CC. Comparisons between traditional medicine and pharmacotherapies for Alzheimer disease: A systematic review and meta-analysis of cognitive outcomes. Int J Geriatr Psychiatry. 2018;33:449-58.

13. Campbell NL, Perkins AJ, Gao S, et al. Adherence and Tolerability of Alzheimer's Disease Medications: A Pragmatic Randomized Trial. J Am Geriatr Soc. 2017;65(7):1497-504. doi:10.1111/jgs.14827 [published Online First: 2017/03/16].

14. Groot C, Hooghiemstra AM, Raijmakers PG, et al. The effect of physical activity on cognitive function in patients with dementia: A meta-analysis of randomized control trials. Ageing Res Rev. 2016;25:13-23. doi: 10.1016/j.arr.2015.11.005 [published Online First: 2015/11/27].

15. Kim K, Han JW, So Y, et al. Cognitive Stimulation as a Therapeutic Modality for Dementia: A MetaAnalysis. Psychiatry Investig. 2017;14(5):626-39. doi:10.4306/pi.2017.14.5.626 [published Online First: 2017/10/19].

16. Dyer SM, Harrison SL, Laver K, et al. An overview of systematic reviews of pharmacological and nonpharmacological interventions for the treatment of behavioral and psychological symptoms of dementia. Int Psychogeriatr. 2018;30(3):295-309. doi: 10.1017/S1041610217002344 [published Online First: 2017/11/17].

17. Liang JH, Lin L, Wang YQ, et al. Non-pharmacological therapeutic strategy options for patients with dementia based on cognitive function-A Bayesian network meta-analysis of randomized controlled trials. Ageing Res Rev. 2019;56:100965. doi:10.1016/j.arr.2019.100965 [published Online First: 2019/11/05].

18. Na R, Yang JH, Yeom Y, et al. A Systematic Review and Meta-Analysis of Nonpharmacological Interventions for Moderate to Severe Dementia. Psychiatry Investig. 2019;16(5):325-35. doi: 10.30773/pi.2019.02.11.2 [published Online First: 2019/05/28].

19. Chou YH, Ton That V, Sundman M. A systematic review and meta-analysis of rTMS effects on cognitive enhancement in mild cognitive impairment and Alzheimer's disease. Neurobiol Aging. 2020;86:1-10. doi:10.1016/j.neurobiolaging.2019.08.020 [published Online First: 2019/11/30].

20. Sharma K. Cholinesterase inhibitors as Alzheimer's therapeutics (Review). Mol Med Rep. 2019;20(2):1479-87. doi:10.3892/mmr.2019.10374 [published Online First: 2019/07/02]. 
21. Scheltens P, Blennow K, Breteler MM, et al. Alzheimer's disease. Lancet. 2016;388(10043):505-17. doi:10.1016/s0140-6736(15)01124-1 [published Online First: 2016/02/28].

22. Assocation AP. Diagnostic and satistical manual of mental disorders: DSM-IV. 4th edn. Washington DC. American Psychiatry Association; 1994.

23. Association AP. Diagnostic and statistical manual of mental disorders: DSM-5. 5thedn Washiton DC. American Psychiatry Association; 2013.

24. Guy M, McKhann. and David S. Knopman ea. The diagnosis of dementia due to Alzheimer's disease: recommendations from the National Institute on Aging-Alzheimer's Association workgroups on diagnostic guidelines for Alzheimer's disease. Alzheimers Dement. 2011;7(3):263.

25. Whiting P, Savovic J, Higgins JP, et al. ROBIS: A new tool to assess risk of bias in systematic reviews was developed. J Clin Epidemiol. 2016;69:225-34. doi:10.1016/j.jclinepi.2015.06.005 [published Online First: 2015/06/21].

26. Auer S, Reisberg B. The GDS/FAST staging system. Int Psychogeriatr 1997;9 Suppl 1:167 - 71. doi: 10.1017/s1041610297004869 [published Online First: 1997/01/01].

\section{Supplementary Files}

This is a list of supplementary files associated with this preprint. Click to download.

- completedPRISMAPchecklist1.docx 